\title{
Exploring the System Dynamics of COVID-19 and Reconfiguration of Emergency Medical Services
}

\author{
Muhammad K. Ali ${ }^{1, *}$, F. Jordan Srour ${ }^{2}$, Nabil Georges Badr ${ }^{3}$, and Abbas Tarhini ${ }^{4}$ \\ ${ }^{1}$ Adnan Kassar School of Business, Lebanese American University, Beirut 1102, Lebanon \\ ${ }^{2}$ Adnan Kassar School of Business, Lebanese American University, Beirut 1102, Lebanon \\ ${ }^{3}$ Higher Institute for Public Health, Saint Joseph University, Beirut 1104, Lebanon \\ ${ }^{4}$ Adnan Kassar School of Business, Lebanese American University, Beirut 1102, Lebanon
}

\begin{abstract}
Emergency Medical Services (EMS) are essential to the medical healthcare landscape. EMS play a crucial role in maximizing the overall expected survival probability of patients with various health emergencies. During the COVID-19 pandemic, peoples' lifestyle changed and their decisions to seek medical assistance were mixed with fear. This affected the type and number of missions that EMS responded to worldwide. Furthermore, COVID-19 affected EMS systems in terms of response protocols and personal protective equipment levels. These changes influenced the EMS' provision of these essential services - fluctuations in demand impacted capacity decisions, the need for PPE altered response times, and lockdowns influenced roadway dynamics. This research focuses on describing and analyzing the performance of a major EMS provider in Lebanon in terms of response time to emergency missions during the COVID-19 pandemic compared to previous years and to the context of EMS in other countries. Results show that the number of calls and number of missions dropped yet, the emergency response time was found to be higher than previous years. The change in response time is most strongly tied to capacity allocation and PPE protocols. This study is an initial step toward exploring EMS system dynamics, with the goal of effectively allocating resources for improved EMS service during periods of heightened uncertainty and variability.
\end{abstract}

\section{Introduction and background}

The World Health Organization (WHO) declared COVID-19 a pandemic, on March 11, 2020 [1]. Consequently, different Emergency Medical Services (EMS) communities experienced varied utilization rates during the early stage of the pandemic. France [2], Copenhagen [3], and Saudi Arabia [4], experienced an increase in call volume while others experienced a decrease in call volume such as the United States [5], Ontario - Canada [6], Italy [7], and Finland [8]. Although EMS communities engage in preparedness training for pandemics and disaster response, the exact protocols required to effectively respond to COVID-19 while minimizing contagion were unanticipated. As a result, the primary service level indicator in

\footnotetext{
*Corresponding author: muhammad.ali@lau.edu
} 
EMS - the response time - was generally longer in communities around the world. Furthermore, as EMS interface with the public, they were subject to the compound impacts of lockdowns and the public's fear of accessing medical intervention making operational decisions for effective service provision even more difficult. For example, a study performed in Canada indicated that overall EMS missions decreased, with a particular drop in motorcycle accidents. On the other hand, the cases of people experiencing overdoses increased [6]. Similarly, a study performed in the United States found that the volume of calls decreased, but the severity of incidents increased [5].

These examples indicate a need to dig deeper into the differential impact of COVID-19 had on EMS systems to learn operational strategies to improve service provision. The abruption of EMS systems in times of COVID-19, in terms of preparedness and response time, provides a great chance to explore and understand EMS system dynamics in "high alert cases". This understanding can help create a baseline from which to enhance EMS systems and develop proper allocation strategies for efficient services in times of high public needs. Our study provides an analysis of the status and performance of one EMS in Lebanon.

As in many developing countries, multiple ambulance agencies exist in Lebanon. The provider supporting this study is the largest, most well-known among those providers and is officially mandated by the Ministry of Health in Lebanon [9]. The role of this agency in maximizing the overall expected survival probability of the patients is well recognized within the healthcare system in Lebanon.

It is important to note that the Lebanese health ecosystem had weathered consecutive crises in Lebanon across the study period. Starting with the Port of Beirut explosion on August 4, 2020, compounded by the economic crisis with an annual inflation rate increase of 133 percent by November 2020, during the height of the pandemic. To exacerbate this challenge, the Port of Beirut explosion destroyed the storage warehouse of all national medical supplies as well as the infrastructure of several hospitals [11]. This deepened crisis increased poverty levels in Lebanon and placed higher demand for an efficient and wellprepared EMS system in Lebanon.

With the first COVID-19 case detected in Lebanon on February 21, 2020 [10], the directors of the EMS developed internal policies to protect their personnel when transporting or dealing with a COVID-19 suspected patient. Such policies included the integration of protocols for personal protective equipment (PPE). While the PPE improved the service safety of the EMS provider, it affected the service speed by introducing a delay in response.

Service science research have looked at ambulance crew scheduling using expected coverage models to establish optimal response time in a dynamically changing setting [21]. Similarly, operations research have analyzed emergency care pathways involving the contribution of multiple actors in the EMS ecosystem, and prepositioning of ambulances to reduce response times [22]. Elsewhere, forecasting models have occupied EMS response research, where an accurate forecast of the demand for emergency medical services (EMS) can help in providing quick and efficient medical treatment and transportation of out-ofhospital patients [23]. Response time may also be vulnerable to planning problems that span demand forecasting, staff scheduling, equipment and resource availability, other environmental factors such as traffic and weather, etc. [24].

In an attempt to learn about the dynamics introduced by COVID-19 pandemic, this paper examines the effect of the pandemic on the response times; exploring what operational levers, the EMS systems may have to enact to avoid significant degradations of service in Lebanon. We observe the capability of reconfiguration of one EMS service ecosystem to respond to disruption caused by the COVID-19 pandemic, through a systemic innovation of the services, adjustments of protocols and calibration of service levels to adapt to the changing constraints [20]. 


\section{Methods}

In order to proceed with this study, data was collected from the systems of a major EMS provider in Lebanon. That data was subsequently cleaned and analyzed through a combination of tools such as PowerBi, Tableau, and Excel. The following subsections provide details on the the data collected, the collection process, and the analysis process.

\subsection{Data collection}

Our study of the performance of EMS in Lebanon is based on primary source data provided by a major EMS provider in Lebanon. The provider implemented a synchronized mission tracking system in 2015 to record data on all missions performed across all of their stations in Lebanon [12]. Data enters the system via the call center where a trained agent logs the mission information following established protocols. The call-taker enters call and mission information into a rules' engine for triage to a recommended response level. Then, based on parameters collected on the location of the patient, correlated with information on availability of ambulances, a team is assigned to the mission. All timestamps for the mission from "call arrived" through to "unit available" along with the chief complaint of the case are directly recorded by the assigned personnel through synchronized electronic devices.

\subsection{Data description}

Our study is based on three datasets.

The first is EMS data collected from the major EMS in Lebanon covering all emergency missions, across Lebanon, received by the central dispatch center from January 2018 through October 2021 [16]. Datasets data have, as each row, one mission served by the EMS. Each mission is described by variables in the columns including the case number, the responding station, responding ambulance vehicle, dispatch type based on the system recommended triage level (Emergency Levels 1, 2, and 3, transport, and high priority transport), chief complaint, mission creation date and time, and arrival to case date and time. These data serve to derive the volume of EMS missions performed on a weekly basis and to investigate the effect of COVID-19 on the volume and response times of the missions (Figure 1). Missions related to the Port of Beirut explosion on August 4, 2020, were removed from the response data due to the high volume of missions in an anomalous and extreme situation. An auxiliary dataset includes the daily number of available EMS teams on duty for the years 2020 and 2021.

The second dataset is a list of governmental COVID-19 precautions and incidents that occurred in Lebanon during the period January 2020 - February 2021. An overlay of this data set on figure 1 shows the potential effect of the precautions made on the EMS response $[10,14]$. 


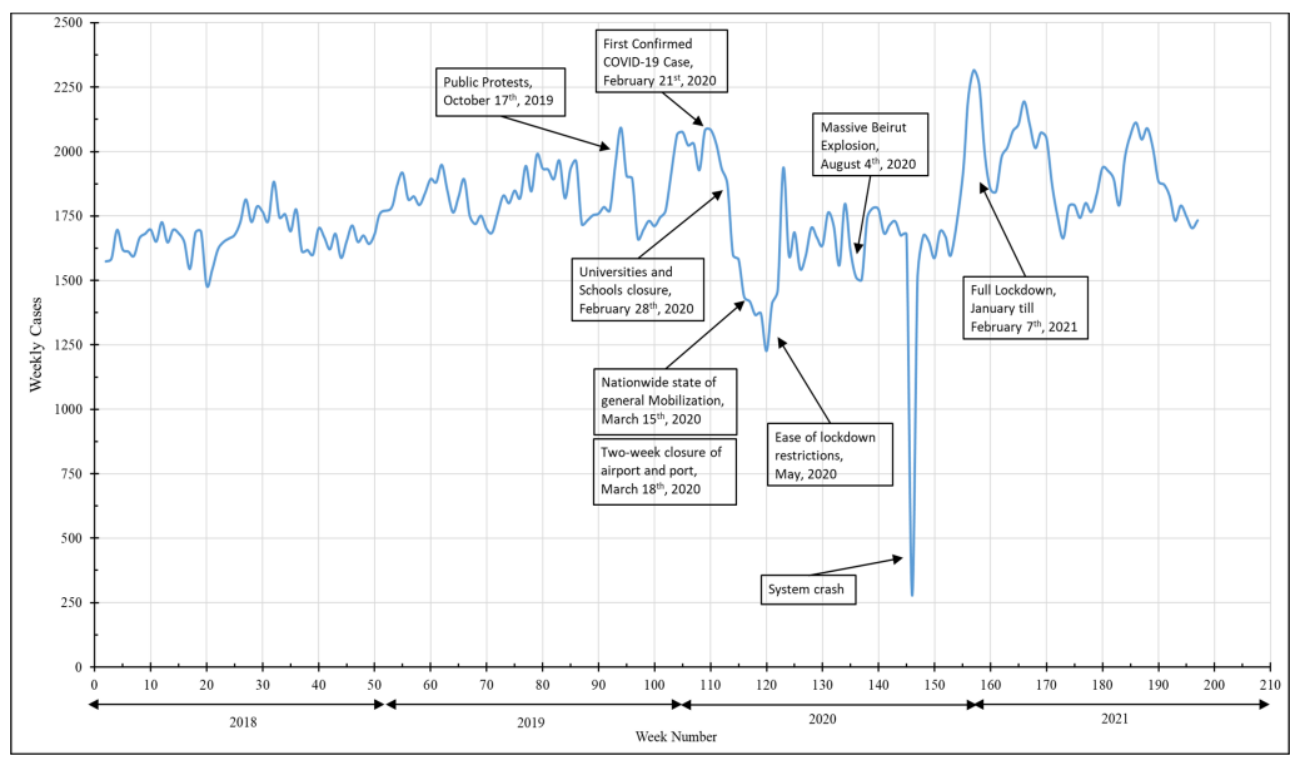

Fig. 1. Weekly Missions 2018-2021

The third dataset is the COVID-19 daily new cases in Lebanon as retrieved from the Johns Hopkins Coronavirus Resource Center. This dataset serves to illuminate the EMS mission variations compared to the spread of COVID-19 in Lebanon [13].

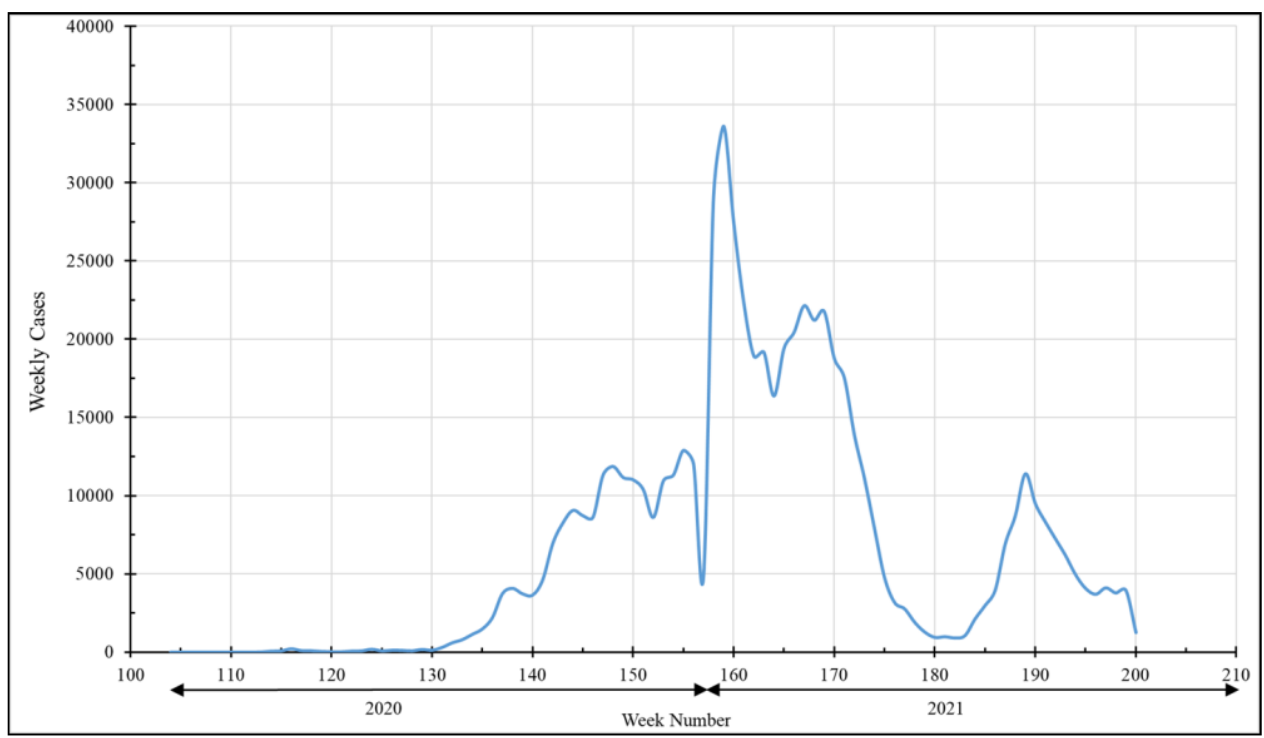

Fig. 2. Weekly New COVID-19 Cases in Lebanon - Source: Johns Hopkins Coronavirus Resource Center

\subsection{Data analysis}

We centered our data analysis on three key axis - (1) Weekly Mission Activity, (2) Resource availability Management (Ambulance \& Teams) and (3) Response Time. 


\subsubsection{Weekly mission activity}

We inspected the weekly mission activity by the EMS provider in the years 2018 to 2021 and include some interesting data visualization in section 3. Weekly EMS missions are graphed for 2018-2021 (Fig. 1) then juxtaposed with a time account of weekly New COVID19 Cases (Fig. 2). We then compile the number of emergency (Section 3. Fig. 4) and transport missions (Section 3. Fig. 5) for each week from 2018 through 2021 and overlay COVID-19 and non-COVID-19 cases in the years 2020 and 2021. The intent is to identify potential correlation between the two data sets.

\subsubsection{Resource management}

In EMS response, key to proper service provision is to have the "right" number of teams on-duty and the resources required such as ambulances and other required accessories, to accommodate the level of demand. The EMS provider in Lebanon had conceived their prehospital care services response as an ecosystem of ambulances, equipment, and trained responders designated as "response teams". Before the pandemic, in 2018 and 2019, weekly EMS missions varied between 1481 and 2092. The number of available teams was around 75 teams a day. These teams were dispatched from a central operations center equipped with an information system that automates the dispatch functions and the related supporting services including volunteer, fleet, equipment, training, and financial management automation [16]. We used data generated from this system as a basis dataset for our analysis.

\subsubsection{Response time}

In EMS, Response Time (RT) is the primary service level indicator used around the world [18]. While there is much debate about the validity of this indicator, it does map well to the expectations of the end-user: an individual who wants care quickly.

While we investigated the overall RT from "Call Received" to "Arrival at Case", we also segmented RT into three time intervals (Fig. 3), in order to examine the potential levers available to EMS providers relative to this metric:

(1) RT1: "call received" to "station notified"; the time from the case creation to the time the station/ambulance was notified, this interval would be most influenced by the number of ambulances available.

(2) RT2: "Station notified" to "departure to case" the time from the notification of the station/ambulance to the departure of the ambulance, influenced by the donning of PPE (an internal protocol influenced by training)

(3) RT3: "departure to case" to "arrival at case" the time from the departure of the ambulance to the arrival to the case, accounting for external factors from roadway conditions that are expected to vary as a function of lockdown periods.

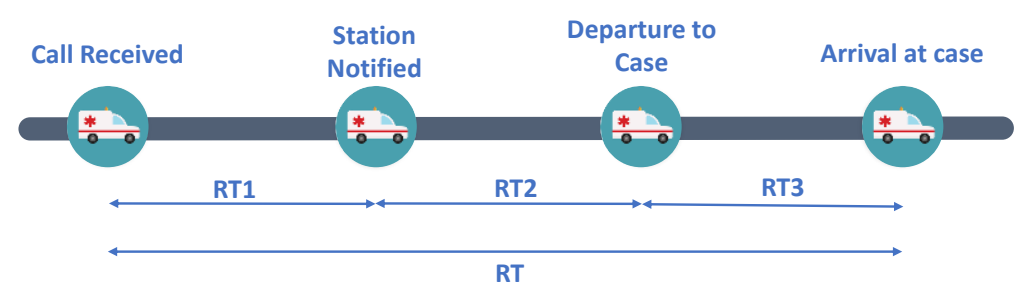

Fig. 3. Response Time Intervals 


\subsection{Data reporting errors and anomalies}

The recording of the "arrival to case" and "case creation" timestamps occurs through the synchronized data management system at the EMS provider. This data management system relies on multiple personnel interfacing with the system through both the computers at the dispatch center and hand-held electronic devices in the field.

As a result, 3 types of data errors have been identified -

(1) Synchronization errors whereby the multiple devices are not properly referenced to the same "standard" clock and

(2) Human errors due to the high intensity environment in which the personnel work.

(2) EMS system availability problems - for instance, the sudden drop in missions in the 44th week is due to a system crash, as reported by the EMS personnel, consistent with the abnormal reporting (Section 3 Fig 3 \& Fig 4.).

Data entry errors manifest in three types of data abnormalities:

(1) no data on the "arrival to a case" due to human error or the nature of the case (e.g. ambulance already on scene during events), making the calculation of response time impossible, one can note that this error rate decreases over the years as the personnel became more familiar with the system;

(2) "arrival to case" timestamps occurring more than 6 hours after the mission was assigned to an ambulance due to human error or extreme latency on the network making the response time illogically long. The noticeable increase in these errors, however relatively still in small numbers, is likely due to COVID-19 cases that require more coordination with the hospitals to handle.

(3) "arrival to case" time stamps occurring before the departure to mission timestamps due to improper system clock synchronization, yielding negative response times, these errors were reduced due to improvements in the technology used;

Table 1 provides a summary table of the missions that were filtered out through this process.

Table 1. Filtered-out Raw Data and Response Times (RT)

\begin{tabular}{|c|c|c|c|c|c|c|}
\hline Period & $\begin{array}{c}\text { Total } \\
\text { Missions }\end{array}$ & $\begin{array}{c}\text { (1) Missions } \\
\text { without RT }\end{array}$ & $\begin{array}{c}\text { (2) Missions } \\
\text { with RT } \\
\text { more than 6 } \\
\text { hours }\end{array}$ & $\begin{array}{c}\text { (3) } \\
\text { Missions } \\
\text { with } \\
\text { negative } \\
\text { RT }\end{array}$ & $\begin{array}{c}\text { Remaining } \\
\text { Missions: RT } \\
\text { between 0 - 6 hrs }\end{array}$ & $\begin{array}{c}\text { Percentage } \\
\text { Filtered-out }\end{array}$ \\
\hline 2018 & 88,612 & 16,405 & 24 & 1,184 & 70,999 & $19.88 \%$ \\
\hline 2019 & 96,327 & 14,025 & 11 & 861 & 81,430 & $15.47 \%$ \\
\hline 2020 & 88,362 & 8,624 & 33 & 2,003 & 77,702 & $12.06 \%$ \\
\hline 2021 & 84,339 & 6,700 & 46 & 461 & 77,132 & $8.55 \%$ \\
\hline
\end{tabular}

\section{Results}

The pandemic and other events in the country have complicated the task on the EMS provider.

\subsection{Weekly mission activity}

Upon inspection of the weekly mission activity by the EMS provider in the years 2018 to 2021 (Figure 1), we find a strong correlation with historical events in the country (Table 2), the spread of the pandemic (Figure 2) and observe the following:

2018 and 2019 show a slight increase in weekly missions with a small increase timed with protests on October 17, 2019, which could be explained by a spike in demand on the resources of the EMS provider. 
The first COVID-19 case was detected on Feb 21 2020, weekly missions dropped significantly from 2085 to 1227 , leading to the lowest number of EMS missions in a week over 2018 to 2021. Emergency missions were low as the number of confirmed COVID-19 cases was still low and panic followed by a lockdown, kept people in their homes. Then COVID-19 related emergency missions began to rise again in May 2020 with the ease of the lockdown and the spread of the pandemic.

In 2021, weekly missions were at around 1700 missions per week until the end of 2020 with the exception of a reported EMS system crash that caused a reporting error.

Jan - Feb 2021 saw a sudden increase of COVID-19 cases, followed by a lockdown. This translated into a record number of missions (2315), then back to an average of 1800 missions / week with a significant variability $(1700-2100)$.

We compile the number of emergency (Fig. 3) and transport missions (Fig. 5) for each week (2018 - 2021) and overlay COVID-19 and non-COVID-19 cases in the years 2020 and 2021. The intent is to identify potential correlation between the two data sets. A key observation here is that the weekly variability of EMS emergency missions follow the variation pattern of COVID-19 cases (Fig. 4) while transport missions seemed loosely correlated (Fig. 5) - as it is apparent that the EMS provider treated COVID-19 cases more as emergency missions, especially in time of surge in the spread of pandemic.

Table 2. Weekly Mission Activity

\begin{tabular}{|c|c|c|}
\hline Period (Week) & Reported Weekly Mission Activity & Potentially Related Events \\
\hline $\begin{array}{l}\text { Oct }-2019 \\
\text { (Week 94) }\end{array}$ & $\begin{array}{l}\text { Spike in demand on the resources of the } \\
\text { EMS provider }\end{array}$ & Protests on October 17, 2019 \\
\hline $\begin{array}{l}\text { Feb-2020 } \\
(\text { Week 112) }\end{array}$ & Missions were at 2100 per week & $\begin{array}{l}1^{\text {st }} \text { COVID-19 Case } \\
\text { (Panic) Feb 21st } 2020\end{array}$ \\
\hline $\begin{array}{l}\text { Feb }-2020 \\
(\text { Week } 113)\end{array}$ & \multirow{2}{*}{$\begin{array}{l}\text { Missions dropped from } 2085 \text { to } 1227 \text { per } \\
\text { week (emergency missions dropped from } \\
\text { around } 1400 \text { missions to around } 1000 \\
\text { missions) }\end{array}$} & $\begin{array}{l}\text { Universities and Schools closure } \\
\text { Feb } 28^{\text {th }} 2020\end{array}$ \\
\hline $\begin{array}{l}\text { Mar - 2020 } \\
(\text { Week 116) }\end{array}$ & & $\begin{array}{l}\text { Nationwide Mobilization ( } 2 \text { week } \\
\text { closure of Port and Airport) }\end{array}$ \\
\hline $\begin{array}{l}\text { May }-2020 \\
(\text { Week } 125)\end{array}$ & $\begin{array}{l}\text { Missions surged back to } 1950 \text { within } 3 \\
\text { weeks }\end{array}$ & Ease of Lockdown \\
\hline $\begin{array}{l}\text { Aug }-2020 \\
(\text { Week 136) }\end{array}$ & $\begin{array}{l}\text { Spike in demand on the resources of the } \\
\text { EMS provider* }\end{array}$ & $\begin{array}{l}\text { Aug } 4^{\text {th }} 2020 \text { - Port of Beirut } \\
\text { explosion }\end{array}$ \\
\hline $\begin{array}{l}\text { Oct }-2020 \\
(\text { Week } 146)\end{array}$ & Reporting error & Reported EMS system crash \\
\hline $\begin{array}{l}\text { Jan - Feb 2021 } \\
\text { (Week 158) }\end{array}$ & $\begin{array}{l}\text { Highest number of missions } 2315-\text { Then } \\
\text { back to an average of } 1800 \text { missions / } \\
\text { week with a significant variability (1700 - } \\
2100 \text { ). }\end{array}$ & $\begin{array}{l}\text { A sudden increase of COVID-19 } \\
\text { cases, followed by a lockdown. }\end{array}$ \\
\hline
\end{tabular}

*Note: The EMS missions related to mission in response to the Port of Beirut explosion on August 4, 2020, were removed from the dataset due to the high volume of missions in an anomalous and extreme situation. 


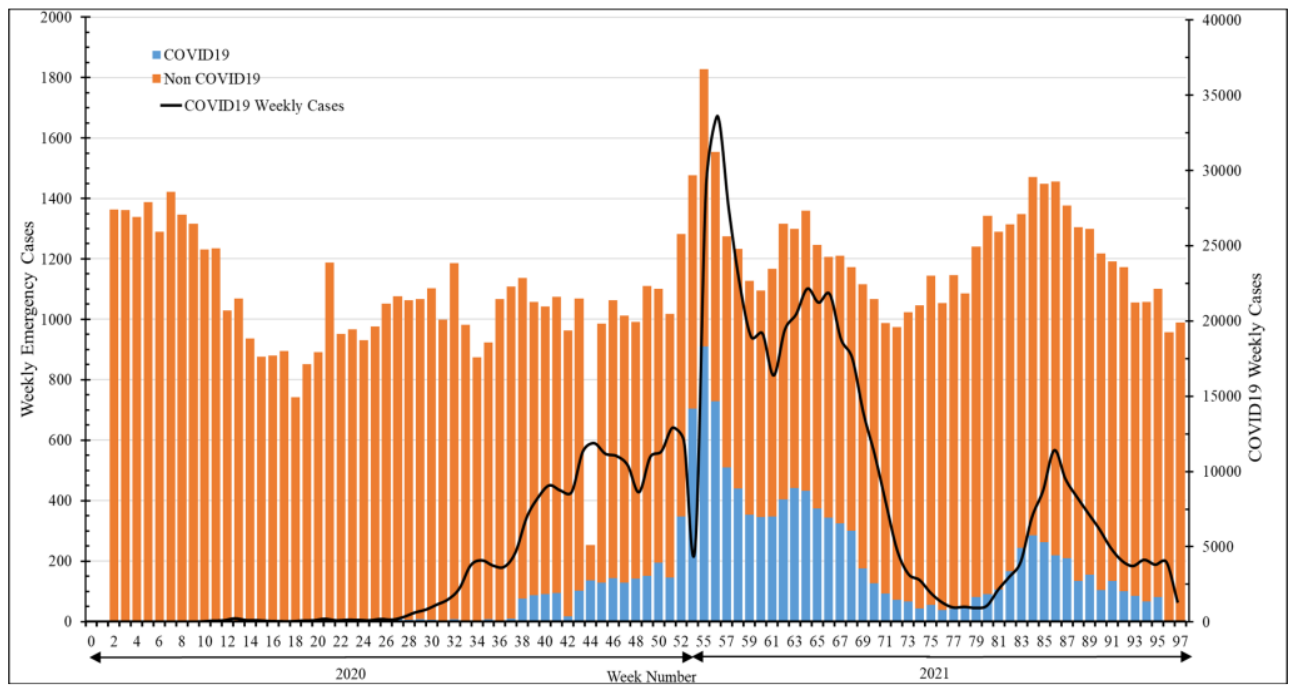

Fig. 4. Weekly Emergency Missions for 2020 and 2021 - COVID-19 and Non COVID-19 Cases

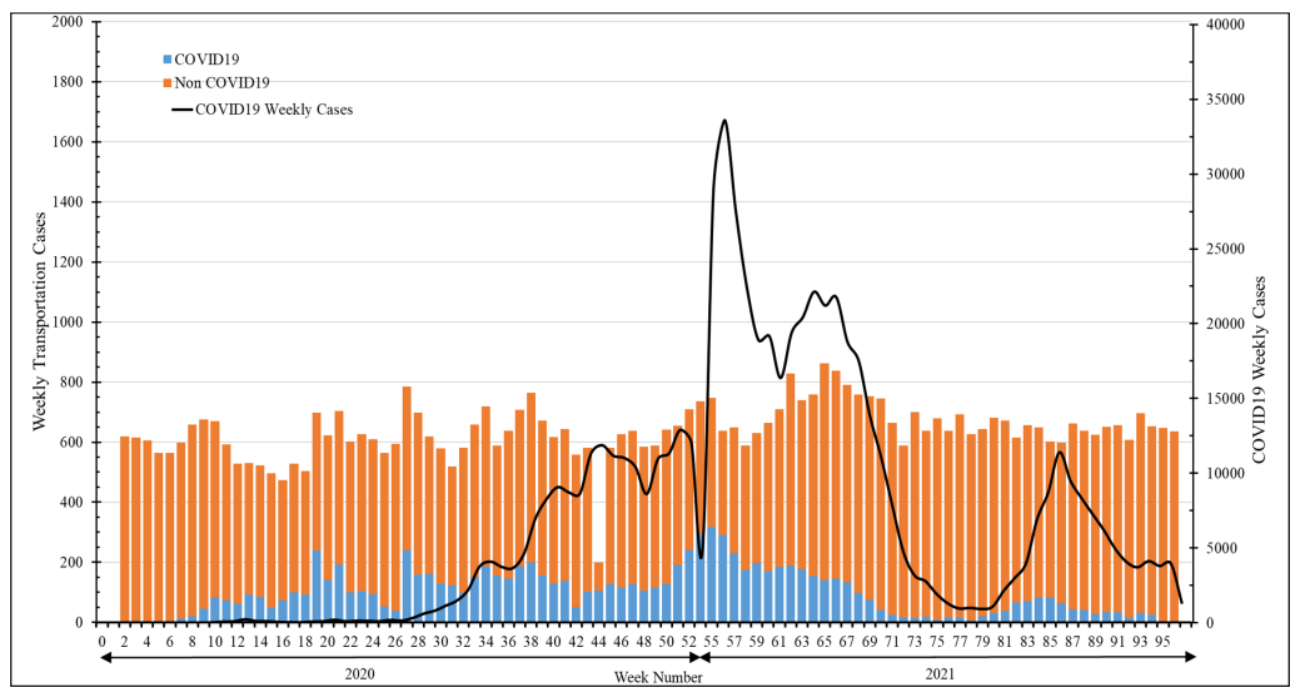

Fig. 5. Weekly Transportation Missions for 2020 and 2021

\subsection{Resource management}

As expected, during the pandemic, the demand increased. What is more remarkable is that the variability in demand was undoubtedly illustrative of a significant difficulty in planning. Fig. 5 shows the variation of weekly missions (left vertical axis) plotted against the number of available teams (right vertical axis), also summarized in Table 3. Before the pandemic, in 2018 and 2019, weekly EMS missions varied between 1481 and 2092. The number of available teams was around 75 teams a day.

In 2020 and 2021, the variation in weekly demand significantly increased to range between 1227 and 2315 missions (Fig. 6).

In the early stages of COVID-19, the number of available teams dipped to almost 50 teams per day, as the EMS provider was ramping up resource to respond to the pandemic with the provisioning of PPE and adjustments to protocols. 
Table 3. Variation of Weekly EMS Missions and Availability of Teams

\begin{tabular}{|c|c|c|}
\hline Period & $\begin{array}{lc}\begin{array}{l}\text { Weekly } \\
\text { missions }\end{array} & \text { EMS }\end{array}$ & Resources available (Teams) \\
\hline $2018-2019$ & $1481-2092$ & 75 (Prior to COVID-19) \\
\hline $2020-2021$ & $1227-2315$ & $\begin{array}{l}\text { Availability dipped to } 50 \text { at first } \\
\text { Increased to } 130 \text { by mid-July } 2020 \\
\text { Then normalized to } 85 \text { (during COVID-19) }\end{array}$ \\
\hline
\end{tabular}

In response to these fluctuations, the EMS provider increased the number of daily available teams to almost 130 by mid-July 2020 (132 ${ }^{\text {nd }}$ week) as evidenced by the bar chart at the bottom of Fig. 6 .

After this significant increase, the level of on-duty teams normalized to 85 to meet the new levels of EMS demand.

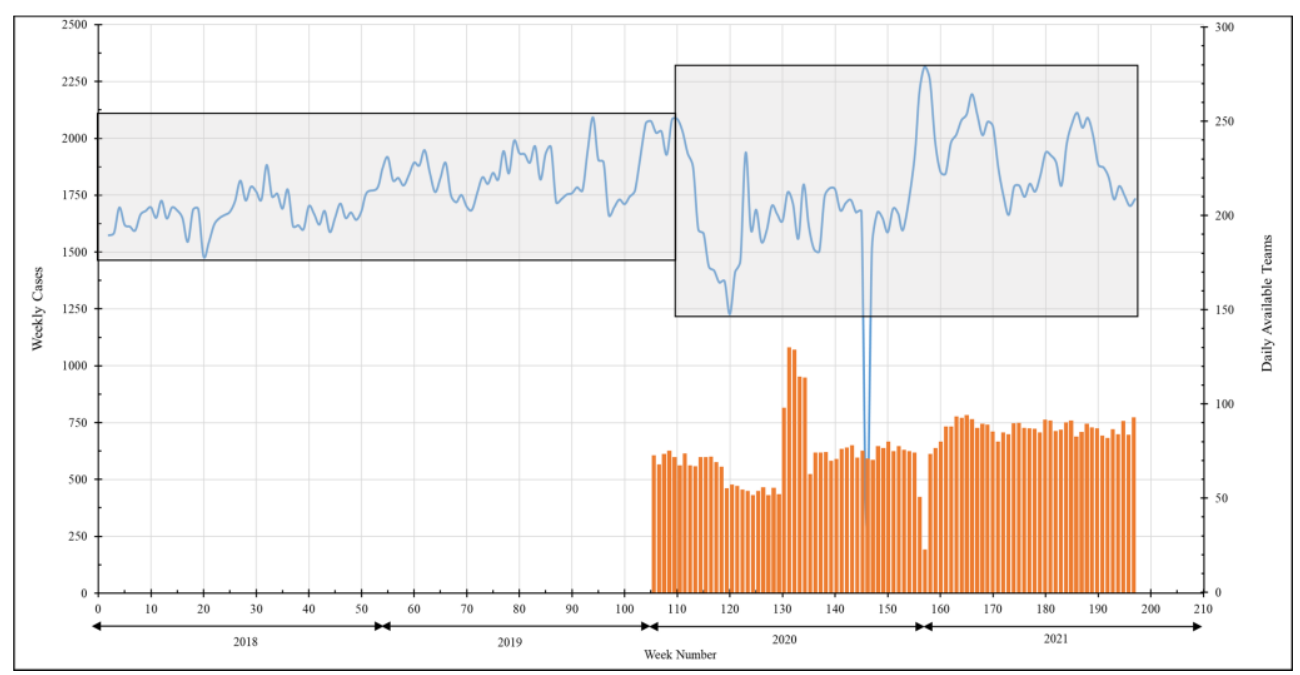

Fig. 6. Variation of Weekly Missions and Available Teams

\subsection{Response time (RT)}

To get a clear indication of the transient fluctuations in actual RT, we plotted RT for the emergency missions only as box and whisker plots across 2018 and 2021. This a means to show the spread and centers of the data set.

The box plot (Fig. 7) shows a clear change of RT for emergency missions overall between Sep 2020 and Apr 2021 and again a small variation in Aug 2021 - this correlates with a spike in COVID-19 cases in Lebanon (Fig. 2). 


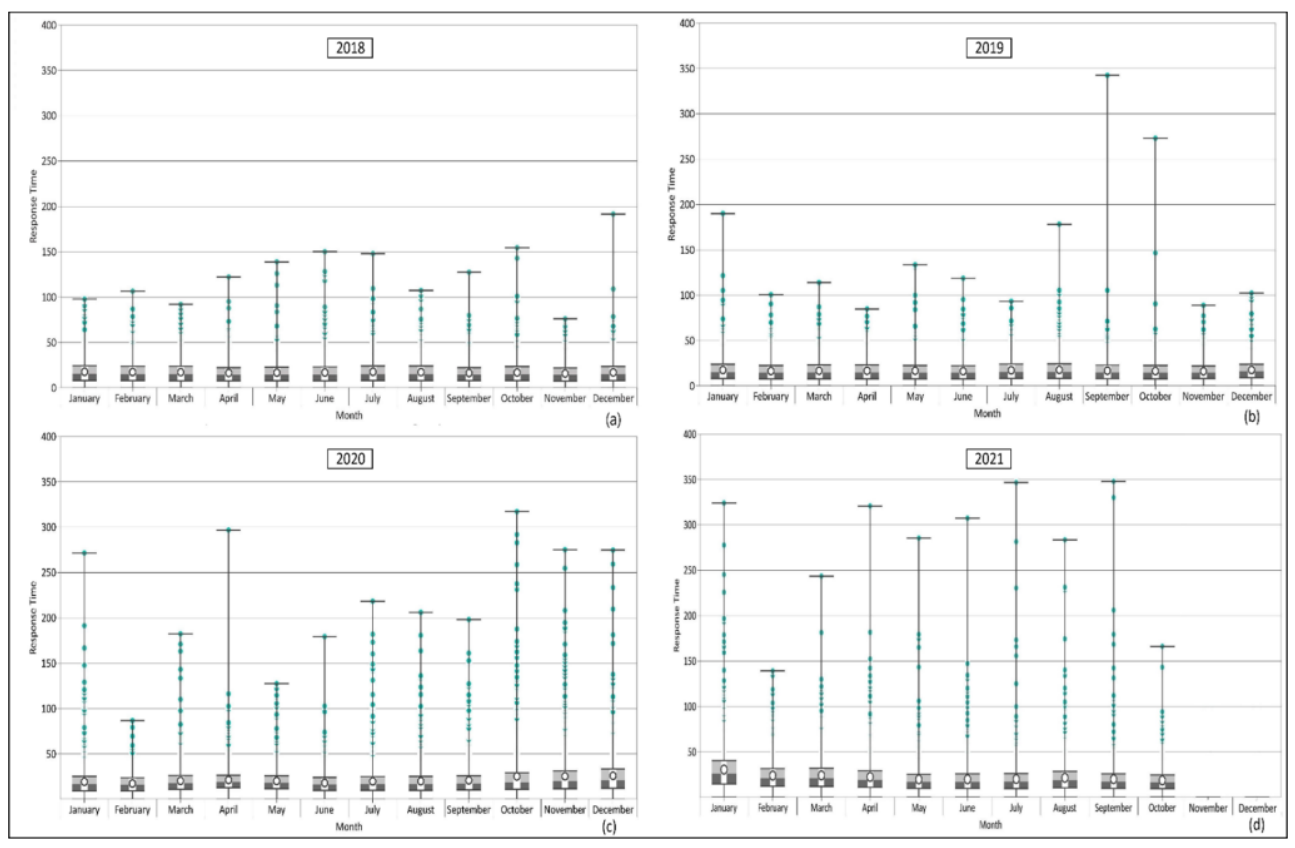

Fig. 7. RT box and plot: 2018-2021 - All Emergency Missions: (a) 2018; (b) 2019; (c) 2020; (d) 2021 Among the emergency COVID-19 related missions, this RT variation is more evident in 2020, at the early stages of the pandemic (Fig. 8).
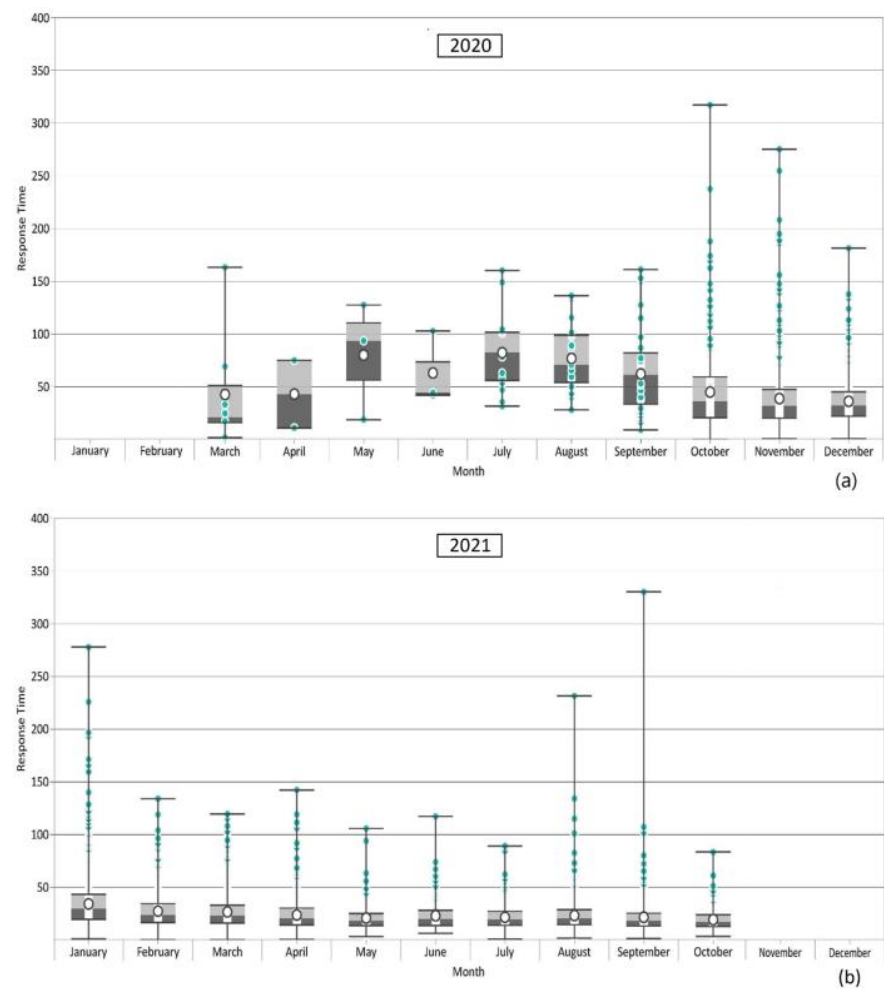

Fig. 8. RT box and plot for Emergency COVID-19 Missions: (a) 2020; (b) 2021 


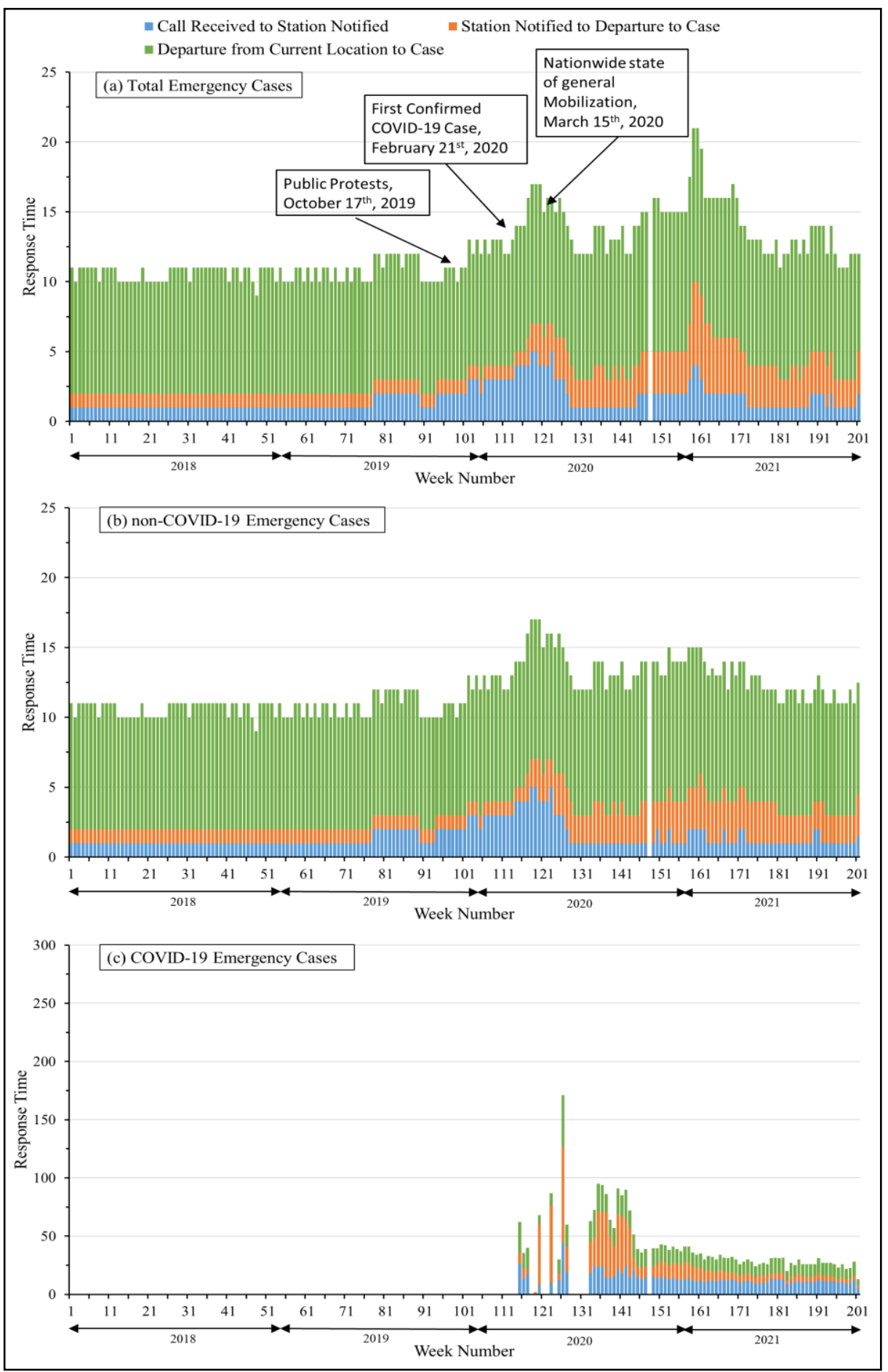

Fig. 9. Median of Segmented Weekly RT of Emergency Missions 2018-2021: (a) Total Emergency Missions; (b) non-COVID-19 Emergency Missions; (c) COVID-19 Emergency Missions 
Furthermore, as indicated in the previous section, we segmented the RT into three time intervals: (1) RT1: "Call received" to "Station notified"; (2) RT2: "Station notified" to "departure to case"; and (3) RT3: "Departure to case" to "arrival at case" the time from the departure of the ambulance to the arrival to the case.

Visuals in Fig. 8 tell the story of how variability in RT1 and RT2 seem to correlate with the variation of COVID-19 cases, while RT3 seem to be less evident.

Until Public Protests Oct 2019, the median response times across all three segments are very consistent with the variation entirely due to external, roadway effects (RT3).

At the start of the protests, RT1 increased due to a sudden increase in the number of missions and the initial limited capacity of the EMS provider.

Similarly, at early stages of COVID-19, the increase in RT2 was largely due to the imposed PPE levels where COVID-19 cases require a longer preparation procedure. While RT1 returned to numbers comparable to the pre-pandemic, RT2, which includes the added preparatory time for departure has remained higher than what it was pre-pandemic.

Nevertheless, the EMS provider was able to normalize the average RT Number of COVID-19 cases spike to 34000 weekly, far exceeded those of 2020 but the number of available teams on duty was increased.

\section{Summary and discussion}

COVID-19 affected service systems and among them are patients and emergency medical services. With a varied EMS call volumes across different countries, and with unexpected protection measures required to respond to COVID-19 cases, the response time of EMS across different communities was generally longer than years preceding COVID-19 [2-8]. In Lebanon, EMS are facing a big challenge in coping with COVID-19 mixed with the consecutive crises occurring in the country [11].

In early COVID-19 stages in Lebanon, the number of weekly missions significantly dropped by around $40 \%$. This observation is in line with the impact seen on the EMS in Pennsylvania [18], for example. However, in general, mixed results in the literature appear across different EMS communities. The drop of EMS missions in Lebanon, followed by a drop in the EMS available teams, resulted in an increase in the median response time (Table 4). The increase is due to the internal factors, where the time to allocate available stations/ambulances increased.

After the drop of EMS missions in early COVID-19 stages in Lebanon, the number of weekly missions changed relative to the number of weekly COVID-19 cases. EMS resources were to adapt to life under the pandemic, adopt new protocols and retool to respond.

Missions dropped at the start with the forced lockdown then surged when the lockdown eased. The EMS had to increase its resources (teams) by more than 50\% within a few weeks. As COVID-19 infections continued to rise to reach a plateau between 7000 - 12000 weekly, the EMS resources had to almost double to keep up with demand to 130 teams. As they learned new skills and adapt to new protocols, they became more efficient - evidenced by the fact that in 2021, the number of COVID-19 cases spiked to 34000 weekly, far exceeding the levels of 2020 but the number of available teams on duty was adapted to 85 teams.

Further, the peak across 2018 to 2021 in weekly EMS missions is observed in early 2021 with the peak of COVID-19 weekly missions, as shown in Fig. 1. The increase of COVID19 cases along with the wide variation in EMS missions resulted in an increase in response times since the EMS internal response was delayed compared to previous years, faced by the disruption of the pandemic. The delay was more likely due to the allocation of available teams and team preparations prior to the mission. The EMS partially controlled the delay by increasing the number of daily available teams and maintained a stabilized response time in terms of internal and external factors in late 2021 (Table 4). 
With that, the EMS was able to recover response times of missions comparable to prepandemic levels (Fig 9) after a transient variability lasting about 18 months.

Table 4. Summative side by side of RT - Emergency Missions; Resource availability; Events

\begin{tabular}{|c|c|c|c|c|c|c|}
\hline & \multicolumn{3}{|c|}{ RT - Emergency Missions (Minutes) } & \multirow[b]{2}{*}{$\begin{array}{c}\text { Resource } \\
\text { Availability } \\
\text { (EMS } \\
\text { Teams) } \\
\end{array}$} & \multirow[b]{2}{*}{$\begin{array}{c}\text { Weekly } \\
\text { Missions }\end{array}$} & \multirow[b]{2}{*}{$\begin{array}{c}\text { Event \& } \\
\text { External } \\
\text { Factors }\end{array}$} \\
\hline Period & $\begin{array}{c}\text { RT } \\
\text { COVID-19 } \\
\text { Missions }\end{array}$ & $\begin{array}{c}\text { RT Non- } \\
\text { COVID- } \\
19 \\
\text { Missions }\end{array}$ & $\begin{array}{c}\text { RT (All } \\
\text { Avg) } \\
\text { Emergency } \\
\text { Missions }\end{array}$ & & & \\
\hline $\begin{array}{c}2018- \\
\text { Oct } 2019\end{array}$ & $\mathrm{~N} / \mathrm{A}$ & N/A & $12-14$ & 75 & $\begin{array}{l}\sim 1481- \\
2092\end{array}$ & $\begin{array}{c}\text { Prior to COVID- } \\
19\end{array}$ \\
\hline $\begin{array}{c}2020 \\
(\mathrm{Jan}-\mathrm{Feb})\end{array}$ & N/A & $10-12$ & 14 & 75 & $\sim 2100$ & $\begin{array}{c}\text { First COVID-19 } \\
\text { case recorded } \\
\text { Feb 21st }\end{array}$ \\
\hline $\begin{array}{l}\text { Mar - } \\
2020\end{array}$ & $\begin{array}{c}60-175 \\
\quad \text { Avg). } \\
\text { Median: } 25\end{array}$ & $14-17$ & 17 & 50 & $\begin{array}{c}\text { Drop 2085 } \\
\text { to 1227 } \\
\text { (emergency } \\
\text { drop 1400 to } \\
\sim 1000 \text { ) }\end{array}$ & $\begin{array}{c}\text { Nationwide } \\
\text { Mobilization (2 } \\
\text { week closure of } \\
\text { Port and Airport) }\end{array}$ \\
\hline $\begin{array}{l}\text { May- } \\
2020\end{array}$ & $\begin{array}{c}\begin{array}{l}90-50 \\
\text { (Avg). }\end{array} \\
\text { Median: } 75\end{array}$ & $15-12$ & 22 & 85 & $\begin{array}{l}\text { Surged to } \\
1950 \text { within } \\
3 \text { weeks }\end{array}$ & $\begin{array}{l}\quad \text { Ease of } \\
\text { Lockdown } \\
\text { COVID cases } \\
\text { rise to } 12000 \\
\text { weekly } \\
\end{array}$ \\
\hline $\begin{array}{c}2020 \\
\text { (Jul - Dec) }\end{array}$ & & & & $\begin{array}{l}\text { Increase to } \\
130 \text { back to } \\
85\end{array}$ & $\begin{array}{l}\sim 1500- \\
1900\end{array}$ & $\begin{array}{c}\text { COVID cases } \\
\text { rise to reach a } \\
\text { plateau between } \\
7000-12000 \\
\text { weekly }\end{array}$ \\
\hline 2021 & $\begin{array}{c}20-25 \\
\text { (Avg). } \\
\text { Median: } 25\end{array}$ & $15-12$ & 12 & 85 & $\begin{array}{l}\text { Peak at } 2315 \\
\text { (Weekly } \\
\text { Record)- } \\
\text { Then back to } \\
\sim 1800 \\
\text { /week with } \\
\text { significant } \\
\text { variability } \\
(1700- \\
2100) .\end{array}$ & $\begin{array}{c}\text { Number of } \\
\text { COVID-19 cases } \\
\text { spike to } 34000 \\
\text { weekly, far } \\
\text { exceeded those } \\
\text { of } 2020 \text { but the } \\
\text { number of } \\
\text { available teams } \\
\text { on duty was } \\
\text { increased. }\end{array}$ \\
\hline
\end{tabular}

\section{Closing remarks}

This work is really an initial step toward exploring EMS system dynamics. Our study investigates the performance of EMS responding to patients in Lebanon in terms of the response time relative to the emergency missions. This article is an initial input regarding the systems dynamics in developing a model that leverages data analytics and response time prediction to intelligently allocate resources for improved EMS performance during such a peculiar emergency.

Patients and EMS are viewed as service systems, which interact to result with patients' lifesaving while having the response time of EMS as one of the most valued in the interacting services [15]. With that, this paper highlights the EMS capability of reconfiguration to innovate the service through learning in terms of value co-creating service interactions. 
The value of service systems was improved by informed value creation from data collected and re-ingested into their systems for better dispatch and decision making, with a focus on dynamic internal and external factors that have been found to affect the systems' performance [16].

The allocation of available teams and the PPE levels set to protect the EMS personnel control the internal factors. The external factor depends on an interaction between emergency facilities location models and transportation networks that include routing and distance to patient case based on the time and location of available ambulance [17]. We do not have data on external factors, as it was not a lever engaged in by the data provider, however, our analysis has shown the potential correlation between the performance of the EMS provider and the variation of COVID-19 cases, the greater potential for panic, governmental decisions on lockdowns, etc.

Thus, we can see the importance of designing a robust service system with operational levers that can be adjusted to respond to dynamic situations. In our case, the improvement in response times was due to changes in the PPE protocols, increased learning for adaptation, training to establish the new protocols along with the operational adjustments to manage the resources variability during the course of the pandemic.

The results of our study provide insight into the tools that EMS providers might adopt to cope with the internal and external implications of system disruption and the importance of learnings from data, used to continually adjust the response capabilities during the pandemic and after. Through early detection of increases in variability of demand, capacity can be readily adjusted to accommodate. In the study, it was noticed that the response time, representing a major value of EMS, was mainly controlled by the increase of daily available teams to accommodate for COVID-19 PPE. For that, the use of optimization to design robust team/ambulance allocation strategies in the face sudden demand fluctuations can serve to enhance service level performance, particularly in terms of response times.

Finally, the ability for the EMS provider to use their information systems and continuously analyze the collected data for innovating their processes and protocols, aiming at keeping response time at the level of service performance, adjusting levers of protocols, resources, and prioritization to innovate, learn and adapt. A clear example of how Service ecosystems co-create value through the reconfiguration of resources (human and other) data driven decision making for a systematic innovation of the services to adapt to the changing constraints, disruption and uphold their value propositions.

\section{References}

1. WHO World Health Organization. Coronavirus disease 2019 (COVID-19) situation report-51. 2019/situation-reports

2. Y. Penverne, J. Jenvrin, and E. Montassier, EMS dispatch center activity during the COVID-19 containment, The American Journal of Emergency Medicine (2020)

3. T. Jensen et al., Strategies to handle increased demand in the COVID-19 crisis: a coronavirus EMS support track and a web-based Self-Triage system, Prehospital Emergency Care, vol. 25, no. 1, pp. 28-38 (2020)

4. A. Al-Wathinani et al., Increased emergency calls during the COVID-19 pandemic in Saudi Arabia: A national retrospective study, in Healthcare, vol. 9, no. 1: Multidisciplinary Digital Publishing Institute, p. 14 (2021)

5. E. B. Lerner, C. D. Newgard, and N. C. Mann, Effect of the Coronavirus Disease 2019 (COVID-19) pandemic on the US Emergency medical services system: a preliminary report, Academic Emergency Medicine, vol. 27, no. 8, pp. 693-699 (2020) 
6. R. Ferron, G. Agarwal, R. Cooper, and D. Munkley, The effect of COVID-19 on emergency medical service call volumes and patient acuity: a cross-sectional study in Niagara, Ontario, BMC Emergency Medicine, vol. 21, no. 1, pp. 1-8 (2021)

7. M. Lazzerini, E. Barbi, A. Apicella, F. Marchetti, F. Cardinale, and G. Trobia, Delayed access or provision of care in Italy resulting from fear of COVID-19, The Lancet Child \& Adolescent Health, vol. 4, no. 5, pp. e10-e11 (2020)

8. L. Laukkanen, S. Lahtinen, J. Liisanantti, T. Kaakinen, A. Ehrola, and L. Raatiniemi, Early impact of the COVID-19 pandemic and social restrictions on ambulance missions, European journal of public health (2021)

9. E. B. L. El-Jardali F, Faraj Y, El Sayed M, Jabr N, K2P Policy Brief: Strengthening Emergency Medical Services in Lebanon, Knowledge to Policy (K2P) Center (2017)

10. J. Koweyes, T. Salloum, S. Haidar, G. Merhi, and S. Tokajian, COVID-19 Pandemic in Lebanon: One Year Later, What Have We Learnt?, Msystems, vol. 6, no. 2, pp. e00351$21(2021)$

11. M. J. El Sayed, Beirut Ammonium Nitrate Explosion: A Man-Made Disaster in Times of the COVID-19 Pandemic, Disaster medicine and public health preparedness, pp. 1-5 (2020)

12. Tarhini, P. Balozain, and F. J. Srour, Emergency management system design for accurate data: a cognitive analytics management approach, Journal of Enterprise Information Management (2021)

13. E. Dong, H. Du, and L. Gardner, An interactive web-based dashboard to track COVID19 in real time, The Lancet infectious diseases, vol. 20, no. 5, pp. 533-534 (2020)

14. Project Hope. On-the-record updates: the COVID-19 situation in Lebanon. https://reliefweb.int/report/lebanon/record-updates-covid-19-situation-lebanon-21january-2021

15. J. Spohrer, S. L. Vargo, N. Caswell, and P. P. Maglio, The service system is the basic abstraction of service science, in Proceedings of the 41st annual hawaii international conference on system sciences (HICSS 2008) (2008): IEEE, pp. 104-104.

16. R. F. Lusch, S. L. Vargo, and G. Wessels, Toward a conceptual foundation for service science: Contributions from service-dominant logic, IBM systems journal, vol. 47, no. 1, pp. 5-14 (2008)

17. Y. Liu, Y. Yuan, J. Shen, and W. Gao, Emergency response facility location in transportation networks: a literature review, Journal of traffic and transportation engineering (English edition) (2021)

18. Al-Shaqsi, Sultan Zayed Khalifah. Response time as a sole performance indicator in EMS: Pitfalls and solutions. Open access emergency medicine : OAEM vol. 2 1-6. 8 Jan. (2010)

19. Badr, N. G., (February). Could ICT Be Harnessed for Prehospital Emergency Medical Services?. In Proceedings of the International Joint Conference on Biomedical Engineering Systems and Technologies (pp. 269-276). (2016)

20. Maglio, P.P., Spohrer, J. Fundamentals of service science. J. of the Acad. Mark. Sci. 36, 18-20 (2008).

21. Rajagopalan, H. K., Saydam, C., Setzler, H., \& Sharer, E.. Ambulance deployment and shift scheduling: An integrated approach. Journal of Service Science and Management, 4(01), 66. (2011) 
22. Aringhieri, R., Bruni, M. E., Khodaparasti, S., \& van Essen, J. T.. Emergency medical services and beyond: Addressing new challenges through a wide literature review. Computers \& Operations Research, 78, 349-368. (2017)

23. Steins, K., Matinrad, N., \& Granberg, T., (January). Forecasting the demand for emergency medical services. In Proceedings of the 52nd Hawaii International Conference on System Sciences. (2019)

24. Reuter-Oppermann, M., van den Berg, P. L., \& Vile, J. L. Logistics for emergency medical service systems. Health Systems, 6(3), 187-208. (2017). 